\title{
Avaliação das propriedades térmicas de misturas PLA/SEBS com moringa submetidas a degradação em ambiente marinho
}

Evaluation of the thermal properties of PLA/SEBS blends with moringa subjected to degradation in a marine environment

Evaluación de las propiedades térmicas de la mezclas PLA/SEBS con moringa sometida a degradación en un entorno marino

Recebido: 24/02/2021 | Revisado: 03/03/2021 | Aceito: 26/03/2021 | Publicado: 02/04/2021

\author{
Jéssica Camilla da Costa Lima \\ ORCID: https://orcid.org/0000-0002-2831-7136 \\ Universidade Federal de Pernambuco, Brasil \\ E-mail: jessicacclima@hotmail.com \\ Rosmary Nichele Brandalise \\ ORCID: https://orcid.org/0000-0002-4719-6093 \\ Universidade de Caxias do Sul, Brasil \\ E-mail: rnbranda@ucs.br \\ Yêda Medeiros Bastos de Almeida \\ ORCID: https://orcid.org/0000-0003-1041-7144 \\ Universidade Federal de Pernambuco, Brasil \\ E-mail: yeda.oliveira@ufpe.br \\ Tomás Jeferson Alves de Melo \\ ORCID: https://orcid.org/0000-0002-5879-6764 \\ Universidade Federal de Campina Grande, Brasil \\ E-mail: tomas.jeferson@ufcg.edu.br \\ Glória Maria Vinhas \\ ORCID: https://orcid.org/0000-0001-5073-609X \\ Universidade Federal de Pernambuco, Brasil \\ E-mail: gloria.vinhas@ufpe.br
}

\begin{abstract}
Resumo
Neste trabalho investigou-se a influência do teor de SEBS e Moringa Oleífera nas propriedades térmicas de blendas PLA/SEBS e de dois biocompósitos baseados em duas dessas blendas. Foram preparadas duas blendas PLA/SEBS (90/10 e 70/30), dois biocompósitos de PLA/SEBS/MO (90/10/1 e 70/30/1) e um biocompósito de PLA/MO (100/1), através da mistura por fusão numa extrusora de matriz aberta. As amostras para biodegradação foram obtidas por prensagem a quente, sendo posteriormente inseridas em ambiente marinho simulado. A evolução da degradação foi avaliada por meio da termogravimetria e calorimetria exploratória diferencial. Foi observado um aumento da cristalinidade de todas as composições avaliadas, e uma redução da estabilidade térmica após a exposição ao ambiente marinho por períodos prolongados. Essa maior cristalinidade pode estar relacionada ao consumo das regiões amorfas e a menor estabilidade térmica está vinculada com a degradação hidrolítica do PLA, devido a água absorvida pela moringa em pó. A avaliação da variação de massa mostrou que as misturas com SEBS e Moringa, apresentaram uma taxa de degradação equivalente ou inferior aquela reportada para o PLA puro.
\end{abstract}

Palavras-chave: PLA; SEBS; Pó da folha da moringa oleífera; Ambiente marinho simulado.

\begin{abstract}
In this work, the influence of the SEBS and Moringa Oleifera content on the thermal properties PLA/SEBS blends and of two biocomposites based on two of these blends was investigated. Two PLA/SEBS blends (90/10 and 70/30), two PLA/SEBS/MO biocomposites (90/10/1 and 70/30/1), and a PLA/MO (100/1) biocomposite, were prepared by melt mixing in an open die extruder. The samples for biodegradation were obtained by hot pressing, being subsequently inserted in a simulated marine environment. Degradation development has been evaluated through mass variation, thermogravimetry, and differential scanning calorimetry. It was observed an increase in crystallinity of all the evaluated compositions, and a reduction of the thermal stability after the exposure to the marine environment for prolonged periods This higher crystallinity may be related to the consumption of amorphous regions and the lower thermal stability is linked to the hydrolytic degradation of PLA, due to the water absorbed by the moringa powder. The evaluation of the mass variation showed that the mixtures with SEBS and Moringa, presented a degradation rate equivalent or lower to that reported for neat PLA.
\end{abstract}

Keywords: PLA; SEBS; Moringa oleifera leaf powder; Simulated marine environment. 


\begin{abstract}
Resumen
En este trabajo, se investigó la influencia del contenido de SEBS y Moringa Oleifera en la propiedades termales de mezclas PLA/SEBS y de dos biocompuestos basados en dos de estas mezclas. Se prepararon dos mezclas de PLA/SEBS (90/10 y 70/30), dos biocompuestos de PLA/SEBS/MO (90/10/1 y 70/30/1), y un biocompuesto PLA / MO (100/1), mezclando en estado fundido en una extrusora de matriz abierta. Las muestras para biodegradación se obtuvieron mediante prensado en caliente, siendo posteriormente insertadas en un ambiente marino simulado. La evolución de la degradación se evaluó mediante termogravimetría y calorimetría exploratoria diferencial. Se observó un aumento en la cristalinidad de todas las composiciones evaluadas y una reducción de la estabilidad térmica después de la exposición al medio marino por períodos prolongados. Esta mayor cristalinidad puede estar relacionada con el consumo de regiones amorfas y la menor estabilidad térmica está ligada a la degradación hidrolítica del PLA, debido al agua absorbida por la moringa en polvo. La evaluación de la variación de masa mostró que las mezclas con SEBS y Moringa, presentaron una tasa de degradación equivalente o menor a la reportada para PLA puro.
\end{abstract}

Palabras clave: PLA; SEBS; Polvo de hoja de moringa oleifera; Entorno marino simulado.

\title{
1. Introdução
}

A persistência de plásticos no meio ambiente, a escassez de espaço em aterros e as preocupações com as emissões de gases tóxicos durante sua incineração, têm ampliado o interesse quanto ao desenvolvimento de polímeros biodegradáveis a partir de recursos renováveis (Abdelwahab et al., 2012). Biopolímeros e seus respectivos compósitos, têm sido amplamente considerados para uso em aplicações agrícolas, industriais e médicas devido a sua excelente biocompatibilidade e biodegradabilidade (Jia et al., 2020; Merino et al., 2019; Park et al., 2017; Pérez et al., 2019; Verma et al., 2019; Yahya et al., 2020).

Dentre os polímeros renováveis, o poli (ácido lático) (PLA) é conhecido como um polímero biocompatível, bioreabsorvível e biodegradável, atributos estes que tornam-o superior aos polímeros petroquímicos, principalmente do ponto de vista de consumo energético e ciclo de vida (emissão de $\mathrm{CO}_{2}$ ), pois pode ser obtido a partir de recursos naturais (Fortunati et al., 2013). Embora o PLA possua as vantagens acima citadas, existem certas desvantagens em relação ao seu desempenho, como sua baixa resistência ao impacto, baixa resistência ao calor e alta capacidade de sofrer hidrólise (Abdelwahab et al., 2012). Além diso, os produtos gerado com PLA ainda não tiveram seu uso generalizado devido ao seu custo relativamente alto e sua taxa de biodegradação lenta (Chang et al., 2003; Pollet et al., 2003; Ramsay et al., 1993). Como consequência, várias pesquisas têm se concentrado na produção de misturas de polímeros naturais e sintéticos biodegradáveis. A adição de componentes poliolefínicos à polímeros biodegradáveis também tem sido estudada de forma a se obter produtos com melhores propriedades e com menor custo (Balakrishnan et al., 2010; Haque et al., 2011; Li et al., 2011).

Misturas de PLA com elastômeros termoplásticos são um método eficaz para tenacifica-lo e com isso melhorar suas propriedades (Alias \& Ismail, 2019; Bernardes et al., 2019; Krishnan et al., 2018; Tejada-Oliveros et al., 2021; Wang et al., 2018; Yan \& Spontak, 2019). O poliestireno-b-poli(etileno-co-butileno)-b-poliestireno (SEBS) combina com sucesso as suas propriedades elastoméricas com os baixos custos de processamento (Tsou et al., 2015). O SEBS é um polímero composto por blocos de poliestireno e blocos de borracha. O bloco de borracha é composto por etileno/butilenos (EB) sendo eficiente para atuar como amortecedor, absorvendo a energia do impacto e atuando na interrupção da propagação de trincas em um sistema polimérico (Chow et al., 2018). O SEBS está disponível comercialmente e possui excelente termoestabilidade, tornando-o um dos principais candidatos a melhorar a processabilidade e tenacidade do PLA (Jose et al., 2005; Juárez et al., 2011; Qi et al., 2011; Wilkinson et al., 2004).

Lima et al. (2019) avaliaram o efeito do teor de SEBS e do tratamento térmico nas propriedades de bioblendas de poli (ácido lático)/estireno-etileno-butilenestireno (PLA/SEBS). Os resultados de difração de raios-X (XRD) e calorimetria exploratória diferencial (DSC) mostraram que o SEBS não afetou a cristalinidade do PLA antes e após o tratamento térmico, 
sugerindo que ele não atuou como agente nucleante. $O$ tratamento térmico levou à cristalização e aumento da resistência ao impacto da matriz de PLA. As bioblendas PLA/SEBS contendo 5 e $10 \%$ de SEBS apresentaram a maior resistência ao impacto antes e após o tratamento térmico. Os resultados de SEM mostram que as bioblendas PLA/SEBS são imiscíveis, e que as bioblendas contendo 15 e $20 \%$ de SEBS apresentaram os maiores tamanhos de domínios de fase dispersa de SEBS.

Outra forma de se reduzir custos e melhorar a performace dos produtos a base de PLA, é através do uso de fibras naturais pois elas são ecologicamente corretas, totalmente biodegradáveis, abundantemente disponíveis, renováveis, leves e baratas. Além disso, a mistura de fibras naturais com polímeros, podem resultar em uma elevação da resistência específica e do módulo de elasticidade, além de contribuir no aumento da estabilidade térmica das matrizes poliméricas (George et al., 2020; Sanjay et al., 2018; Vigneshwaran et al., 2020). Dentre as fibras naturais, a moringa oleifera é considerada uma das plantas mais úteis do mundo, pois quase todas as suas partes podem ser utilizadas na alimentação, na medicina tradicional e para fins industriais (Alegbeleye, 2018; Falowo et al., 2018; Gupta et al., 2018; Liu et al., 2018; Valenga et al., 2019).

Mishra e Sinha (2020) aplicaram folhas de moringa oleifera como carga reforçante em compósitos epóxi para aplicações semi-estruturais. Seus compósitos apresentaram maior resistência à água, e maior resistência à tração e flexão, em comparação com o epóxi puro, para uma fração de carga de $20 \%$. Pode-se concluir que a utilização das folhas de moringa é uma ótima alternativa as cargas sintéticas, podendo ser facilmente aplicada em demandas semi-estruturais. Além disso, seu uso aumenta as chances de eliminação de plantas não lenhosas intrusas e destrutivas.

A literatura tem mostrado que a fragilidade e o custo são os principais parâmetros que limitam a comercialização e aplicação generalizada do PLA (Dias \& Chinelatto, 2019; Hakim et al., 2017). Além disso, sabe-se que apesar da biodegradação do PLA ser bastante estudada, ainda não está clara a influência da adição de simultânea de cargas naturais e poliolefinas, na biodegradação do PLA em ambiente marinho simulado. A partir dessa contribuição o uso desses materiais será mais seguro, principalmente em aplicações de uso final (Muniyasamy et al., 2016).

Dentre as ferramentas que podem ser utilizadas estão as técnicas de análise térmica de termogravimetria (TG) e calorimetria exploratória diferencial (DSC). A análise termogravimétria é uma técnica amplamente utilizada para avaliar a estabilidade térmica de misturas e compósitos poliméricos (Gabbott, 2008). A determinação de parâmetros cinéticos como energia de ativação associada à degradação térmica é uma ferramenta importante na determinação da cinética de decomposição térmica de polímeros e compósitos poliméricos (Dhar \& Katiyar, 2017; Mittal et al., 2020). A análise de DSC tem sido aplicada para monitorar mudanças na cristalinidade com base nas mudanças de temperatura de transição vítrea $\left(\mathrm{T}_{\mathrm{g}}\right)$, temperatura de cristalização $\left(T_{c}\right)$, temperatura de fusão $\left(T_{m}\right)$ e calor de fusão (DH) (Capitain et al., 2020). Além disso, pesquisas anteriores tem demonstrado que as diferenças na localização do pico e na largura dos perfis DSC são bons indicadores do nível de degradação da amostra (Iovino et al., 2008). Isso mostra a importância de se aplicar técnicas de análise térmica para se avaliar a biodegradação de materiais poliméricos.

Este artigo tem como objetivo avaliar as alterações nas propriedades térmicas de blendas de PLA/SEBS e biocompósitos com MO quando expostas a um ambiente marinho simulado nos intervalos de 0, 40, 80 e 160 dias. Variou-se a concentração de SEBS (10 e 30\% em massa) e fixou-se a concentração de moringa (1pcr) nos biocompósitos. Os filmes utilizados foram produzidos por meio da mistura por fusão, aplicando-se as técnicas extrusão e moldagem por compressão. A evolução da degradação foi avaliada por meio das análises de termogravimetria, calorimetria exploratória diferencial e perda de massa. 


\section{Metodologia}

\subsection{Materiais}

Poli (ácido láctico) (PLA), Ingeo ${ }^{\circledR}$ 2003D, NatureWorks, MFI $=6 \mathrm{~g} / 10 \mathrm{~min}$, foi fabricado pela NatureWorks (Nebraska, USA) e fornecido pela Cargill. O copolímero tribloco de estireno/etileno-butileno/estireno (SEBS), MFI = 1523g/10 min, Kraton тм G1726 M, foi fabricado e fornecido pela Kraton. O pó das folhas de moringa foi fornecido pela BIOVEA Natural Foods.

\subsection{Métodos}

Inicialmente o PLA e a moringa foram secos em uma estufa Quimis, modelo Q-317 B252, por 12 e $4 \mathrm{~h} \mathrm{a} 60{ }^{\circ} \mathrm{C}$, respectivamente. Após essa etapa realizou-se a pré-mistura manual do SEBS, PLA e Moringa, obedecendo as frações descritas na Tabela 1. As pré-misturas foram alimentadas em uma extrusora dupla-rosca, modelo MH-COR-20-32-LAB, fabricado pela MH Equipamentos Ltda, SP, Brasil, com as especificações L/D=32, L= $640 \mathrm{~mm}$ e D= $20 \mathrm{~mm}$. Aplicou-se uma velocidade de $150 \mathrm{rpm}$, obedecendo o seguinte perfil de temperatura da matriz até o funil: 100,110,120,140,160,175, 175, 175, 165, $155,155,60^{\circ} \mathrm{C}$. Os chips confeccionados foram produzidos com matriz aberta e posteriormente triturados em um moinho de facas da marca MARCONI modelo MA 580.

A moldagem por compressão para formação dos filmes $(10 \times 10 \mathrm{~cm} \times 2 \mathrm{~mm})$ foi realizada em uma prensa SCHULZ PH5 15 t, (Brasil), por $5 \mathrm{~min}$, na temperatura de $170^{\circ} \mathrm{C}$ e pressão de 8 toneladas. Após a prensagem, as placas foram colocadas para resfriar com pressão de 8 ton. em prensa com circulação de água. A partir de cada placa foram confeccionadas 7 amostras para posterior exposição em ambiente marinho simulado (AMS). A Tabela 1 detalha a codificação utilizada para descrever cada uma das composições utilizadas.

Tabela 1 - Codificação do estudo e descrição da composição das bioblendas.

\begin{tabular}{c|c|c|c}
\hline \multicolumn{3}{c|}{ Composições } & \multirow{2}{*}{ Nomenclatura } \\
\hline \multirow{2}{*}{ PLA } & SEBS & Moringa & PLA \\
\hline 100 & 0 & 0 & PLA/SEBS 90/10 \\
\hline 90 & 10 & 0 & PLA/SEBS 70/30 \\
\hline 70 & 30 & 0 & PLA/MO 100/1* \\
\hline 100 & 0 & 1 & PLA/SEBS/MO 90/10/1* \\
\hline 90 & 10 & 1 & PLA/SEBS/MO 70/30/1* \\
\hline 70 & 30 & 1 & \\
\hline
\end{tabular}

* partes 100 por massa de resina. Fonte: Autores.

Os corpos de prova para análise da degradação em ambiente marinho foram dispostos em um aquário da Universidade de Caxias do Sul - UCS-Aquarium/Brasil como apresentado na Figura 1. O aquário tinha as seguintes dimensões: volume de $250 \mathrm{~L}$, largura de $58 \mathrm{~cm}$, comprimento de $110 \mathrm{~cm}$, altura de $50 \mathrm{~cm}$. Foi equipado com 7 lâmpadas (3 brancas, 2 azuis, e duas lilás), equivalentes à $250 \mathrm{w}$, simulando os raios ultravioletas (UV). As condições do ambiente marinho foram: temperatura da água entre $24,5-27^{\circ} \mathrm{C}, \mathrm{pH}$ entre $8,2-8,4$. Além disso, o aquário possuía três peixes sendo um da espécie peixe anjo rainha e os outros dois peixes donzela three stripes e corais Ricordea roxa (Actinodiscus sp), Astrea (Astrea proebia). O controle das condições ideais do ambiente marinho simulado foi realizado por profissionais técnicos do UCS-Aquarium de acordo com o 
monitoramento semanal realizado no sistema que contém formas de vida tais como peixes, corais, crustáceos e algas. As retiradas das amostras dos corpos de prova do estudo foram feitas após 40, 80 e 160 dias de exposição.

Figura 1 - Foto das amostras no ambiente marinho simulado apontadas com as setas amarelas, a) 0 dias e b) após 160 dias de exposição.

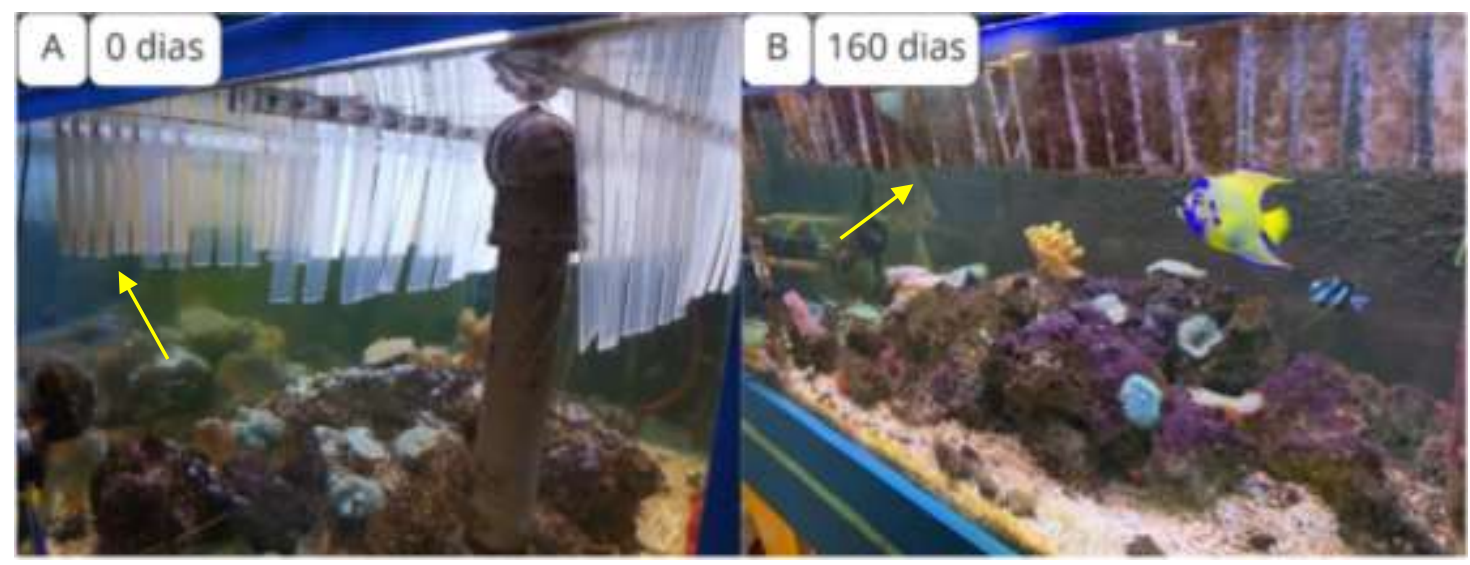

Fonte: Autores.

É importante lembrar que a metodologia científica utilizada para a produção dos corpos de prova que foram posteriormente caracterizados, é de natureza quantitativa (Pereira et al., 2018). Isso acontece porque trabalhou-se com quantidades pré-determinadas de cada componente (PLA, SEBS, Moringa Oleifera), e aplicou-se parâmetros de processamento específicos (temperatura, velocidade da rosca, tempo de prensagem, e etc). Em seguida foram coletados dados de natureza numérica por meio do uso de medições de grandezas por meio das técnicas de caracterização. A partir dai pode-se inferir a respeito do comportamento dos materiais produzidos.

\subsection{Caracterizações}

\subsubsection{Calorimetria Exploratória Diferencial}

As análises de DSC foram realizadas em um calorímetro diferencial de varredura da marca Shimadzu, modelo DSC60 (Japão). Foram aplicadas duas etapas de aquecimento e uma etapa de resfriamento. As amostras foram aquecidas de 23 à $200{ }^{\circ} \mathrm{C}$, resfriadas de 200 à $23^{\circ} \mathrm{C}$ e novamente aquecidas de 23 à $200^{\circ} \mathrm{C}$. Foi utilizada uma taxa de aquecimento de $10^{\circ} \mathrm{C}$.min 1, sob $\mathrm{N}_{2}$ com vazão de 50 mL.min-1. O grau de cristalinidade, com base nos termogramas de DSC, do PLA puro e do PLA presente nas blendas foi calculado de acordo com a equação 1 (Zhang et al., 2003).

$$
X_{c}=\frac{\Delta H_{m}}{\Delta H_{m}^{0} *(1-x)} \times 100 \%
$$

Onde $\Delta \mathrm{Hm}$ (J.-g $\mathrm{g}^{-1}$ do polímero) é a entalpia de fusão e X é a porcentagem em massa de SEBS. $\Delta \mathrm{H}_{m}^{0}$ é a entalpia de fusão de PLA hipoteticamente 100\% cristalino, sendo o valor $93 \mathrm{~J} \mathrm{~g}^{-1}$ (Nehra et al., 2018b) um valor fixo.

\subsubsection{Análise de termogravimetria}

Em um equipamento da marca SHIMADZU, modelo TGA-50 (Japão), foi realizada a análise de TG. As amostras foram aquecidas em uma faixa de temperatura de 10 à $810^{\circ} \mathrm{C}$, com uma taxa de aquecimento de $10^{\circ} \mathrm{C}$.min ${ }^{-1}$ e fluxo de 50 $\mathrm{mL}$.min ${ }^{-1}$ de nitrogênio $\left(\mathrm{N}_{2}\right)$. Avaliou-se a variação a influência da adição do SEBS e Moringa ao PLA, assim como do tempo 
de exposição na estabilidade térmica das composições avaliadas.

\subsubsection{Percentagem de massa residual}

A biodegradação das amostras removidas do ambiente marinho simulado, nos diferentes tempos de exposição, foi avaliada por meio análise da massa residual em \%, como descrito na Equação 2.

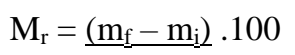

$\mathrm{m}_{\mathrm{i}}$

Onde:

$\mathrm{M}_{\mathrm{r}}$ é a massa residual (\%);

$\mathrm{M}_{\mathrm{f}}$ é a massa final da amostra coletada;

Limpo e seco (g) e mi é a massa inicial do filme, limpo e seco, antes da biodegradação (g).

\section{Resultados e Discussão}

\subsection{Calorimetria Exploratória Diferencial (DSC)}

Pode-se observar na Figura 2, a qual apresenta os valores de cristalinidade do PLA, das suas blendas e dos seus compósitos com moringa, que após 160 dias de exposição ao ambiente marinho simulado, ocorreu aumento do grau de cristalinidade para todas as amostras, como resultado do ataque às regiões amorfas do PLA, de tal forma que o restante da amostra passou a apresentar maior grau de cristalinidade como resultado da perda de integridade da matriz e comprometimento dos segmentos cristalinos restantes (Pelegrini et al., 2016). A presença constante da água advinda do ambiente marinho simulado, provavelmente acelerou o processo de degração hidrolítica do PLA, reduzindo sua massa molar e consequentemente aumentando a fração cristalina.

Figura 2 - Valores de cristalinidade obtidos a partir do DSC do PLA, das blendas PLA/SEBS e dos seus compósitos com moringa.

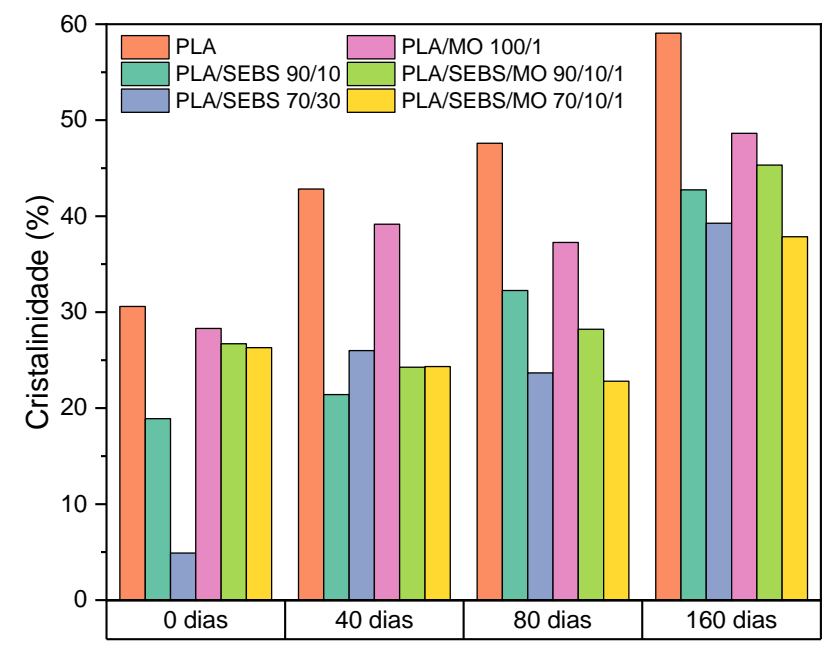

Fonte: Autores.

Para a composição PLA/MO, foi observado aumento na cristalinidade, atribuído ao fato de que a presença da 
moringa oleífera pode ter possibilitado uma maior absorção de água, ampliando o acesso à matriz, e consequentemente facilitando a ação de micro-organismos, com comprometimento dos segmentos amorfos, e posterior aumento da cristalinidade (Palsikowski et al., 2018).

Após a remoção do histórico térmico, os dados de DSC da segunda varredura de aquecimento foram obtidos para todas as amostras de PLA, para as blendas de PLA/SEBS (90/10 e 70/30) e biocompósitos de PLA/SEBS/MO (90/10/1 e 70/30/1), para diferentes tempos de exposição ao ambiente marinho simulado, como mostrado na Tabela 2. Comparando os valores da temperatura de fusão cristalina $\left(\mathrm{T}_{\mathrm{m}}\right)$ das composições de 0 dias com tempos de exposição mais avançados, pode-se perceber que seus valores permaneceram praticamente inalterados. Em relação à temperatura de transição vítrea $\left(\mathrm{T}_{\mathrm{g}}\right)$ conclui-se que a adição de 10 e $30 \%$ em massa de SEBS ao PLA levou a um aumento dessa propriedade nas blendas $\left(3^{\circ} \mathrm{C}\right)$, mas o tempo de exposição não afetou a $T_{g}$ de nenhuma das composições.

Tabela 2 - Transições e características térmicas dos materiais estudados com 0, 40, 80 e 160 dias de exposição ao ambiente marinho simulado.

\begin{tabular}{|c|c|c|c|c|c|c|}
\hline Dias & Composições & $\begin{array}{c}\mathrm{T}_{\mathrm{g}} \\
\left({ }^{\circ} \mathrm{C}\right) \\
\end{array}$ & $\begin{array}{c}\mathrm{T}_{\mathrm{c}} \\
\left({ }^{\circ} \mathrm{C}\right)\end{array}$ & $\begin{array}{c}\mathrm{T}_{\mathrm{m}} \\
\left({ }^{\circ} \mathrm{C}\right)\end{array}$ & $\begin{array}{c}\Delta \mathrm{H}_{\mathrm{m}} \\
\left(\mathrm{J} \cdot \mathrm{g}^{-1}\right)\end{array}$ & $\begin{array}{c}\mathrm{X}_{\mathrm{C}} \\
(\%) \\
\end{array}$ \\
\hline \multirow{6}{*}{0} & PLA & 66,8 & 102,2 & 171,5 & 28,5 & 30,6 \\
\hline & PLA/SEBS 90/10 & 70,3 & 106,2 & 170,9 & 15,9 & 18,9 \\
\hline & PLA/SEBS 70/30 & 69,1 & 110,4 & 171,8 & 16,2 & 24,9 \\
\hline & PLA/MO 100/1* & 68,7 & 100,8 & 171,2 & 26,4 & 28,3 \\
\hline & PLA/SEBS/MO 90/10/1* & 68,2 & 106,9 & 172,1 & 22,1 & 26,7 \\
\hline & PLA/SEBS/MO 70/30/1* & 69,9 & 113,2 & 172,3 & 17,1 & 26,3 \\
\hline \multirow{6}{*}{40} & PLA & 65,5 & 101,7 & 169,7 & 34,8 & 42,8 \\
\hline & PLA/SEBS 90/10 & 68,8 & 106,5 & 171,0 & 17,9 & 21,4 \\
\hline & PLA/SEBS 70/30 & 69,1 & 110,8 & 171,6 & 16,9 & 26,0 \\
\hline & PLA/MO 100/1* & 65,9 & 102,2 & 169,8 & 36,4 & 39,2 \\
\hline & PLA/SEBS/MO 90/10/1* & 70,1 & 110,5 & 171,6 & 20,3 & 24,3 \\
\hline & PLA/SEBS/MO 70/30/1* & 71,0 & 113,9 & 171,9 & 15,9 & 24,3 \\
\hline \multirow{6}{*}{80} & PLA & 65,7 & 101,4 & 169,2 & 44,3 & 47,6 \\
\hline & PLA/SEBS 90/10 & 69,9 & 107,2 & 173,0 & 27,0 & 32,3 \\
\hline & PLA/SEBS 70/30 & 68,7 & 110,2 & 171,5 & 15,4 & 23,7 \\
\hline & PLA/MO 100/1* & 67,7 & 102,3 & 170,1 & 34,7 & 37,3 \\
\hline & PLA/SEBS/MO 90/10/1* & 67,1 & 109,0 & 171,2 & 23,6 & 28,2 \\
\hline & PLA/SEBS/MO 70/30/1* & 67,6 & 114,9 & 171,2 & 14,9 & 22,8 \\
\hline \multirow{6}{*}{160} & PLA & 65,2 & 101,9 & 169,4 & 54,9 & 59,1 \\
\hline & PLA/SEBS 90/10 & 68,0 & 104,8 & 170,9 & 35,8 & 42,7 \\
\hline & PLA/SEBS 70/30 & 69,6 & 107,7 & 171,4 & 25,6 & 39,3 \\
\hline & PLA/MO 100/1* & 67,2 & 103,2 & 169,2 & 45,2 & 48,6 \\
\hline & PLA/SEBS/MO 90/10/1* & 68,9 & 108,2 & 169,4 & 37,9 & 45,3 \\
\hline & PLA/SEBS/MO 70/30/1* & 72,3 & 113,9 & 171,0 & 24,7 & 37,9 \\
\hline
\end{tabular}

* partes por 100 por massa de resina. Fonte: Autores.

Assim, quando a influência das diferentes composições foi avaliada, percebeu-se que a adição de SEBS aumentou a $T_{\mathrm{g}}$, em comparação com o PLA puro. Esta mudança para temperaturas mais altas sugere aumento da restrição de cadeia, 
presumivelmente devido a interações interfaciais entre as fases dos polímeros que dificulta o relaxamento molecular (Imre et al., 2013). No entanto, não foi observado diferença significativa na $T_{\mathrm{g}}$ das blendas PLA/SEBS 90/10 e PLA/SEBS 70/30, bem como entre os biocompósitos com moringa (Tabela 2). A adição de SEBS ou moringa não afetou a $T_{m}$. Em relação as entalpias de fusão $(\Delta \mathrm{Hm})$, pode-se observar a redução de seus valores com o aumento da fração SEBS, para as blendas e biocompósistos.

Em relação à temperatura de cristalização $\left(T_{c}\right)$, esse aumento foi de até $10^{\circ} \mathrm{C}$, quando comparamos a composição de PLA com a composição PLA/SEBS/MO 70/30/1. Além disso, a presença de SEBS e Moringa contribuíram ainda mais para esses aumentos, devido ao aumento da estabilidade térmica desses componentes (TSOU et al., 2015). O SEBS é um copolímero amorfo, que quando presente em frações elevadas, pode reduzir a cristalinidade do PLA. Já as partículas de Moringa atuaram como enchimentos, restringindo o movimento molecular da matriz (DOU et al.,) dificultando a cristalização do PLA. No entanto, quando a moringa foi incorporada na mistura, observou-se um maior grau de cristalinidade, em comparação com as mesmas composições sem sua presença, o que pode ser explicado pelo aumento da nucleação e crescimento de esferulitos (Tokoro et al., 2008; Wunderlich et al., 2012).

\subsection{Termogravimetria (TG)}

Várias temperaturas relacionadas à estabilidade térmica foram determinadas a partir dos dados de termogravimetria: $T_{10}, T_{50}$ e $T_{95}$, sendo definidas como as temperaturas onde 10,50 e $95 \%$ da massa total foi volatilizada, respectivamente. Dessa forma, a temperatura $\mathrm{T}_{10}$ fornece uma boa ideia da estabilidade térmica, pois identificar o começo da decomposição térmica muito próximo de 0 é muito difícil já que baixas variações de conversão nessa zona correspondem a altas variações de temperatura (Carrasco et al., 2010; Carrasco et al., 2008).

Com a mudança de 10 para $30 \%$ de SEBS houve um pequeno aumento na estabilidade térmica do PLA, o qual pode estar relacionado a maior estabilidade térmica do SEBS (Nehra et al., 2018a), o qual pode ser observado por meio da Figura 3 onde estão expostas as curvas termogravimétricas das blendas PLA/SEBS e dos seus compósitos com moringa. Após 160 dias de exposição ao ambiente marinho foi observada uma redução na estabilidade térmica proporcional a concentração de SEBS e de moringa oleífera (de até $22^{\circ} \mathrm{C}$ para a composição PLA/SEBS/MO 70/30/1). Esta redução na estabilidade térmica pode ter se dado devido à ação de enzimas microbianas que podem ter causado clivagem ou alteração de ligações (Soni et al., 2009).

Figura 3 - Curvas TGA das blendas PLA/SEBS e dos seus compósitos com moringa.

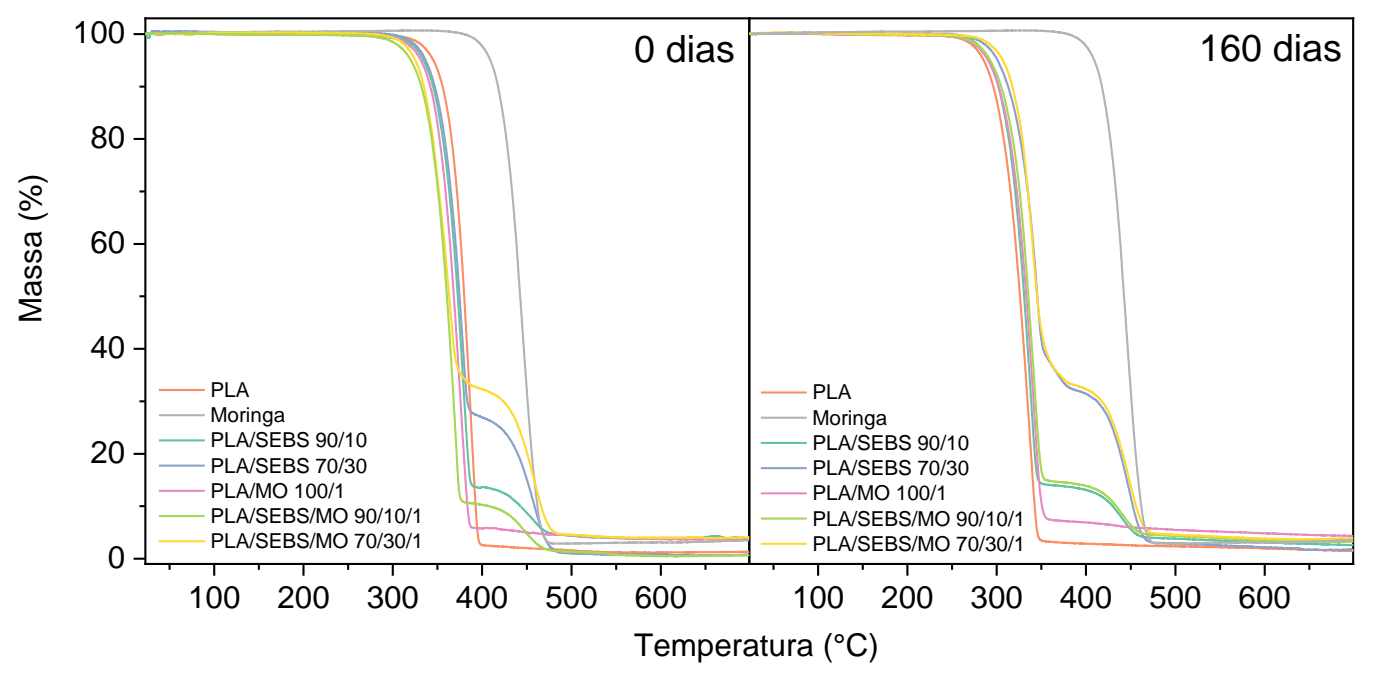

Fonte: Autores. 
Logo, pode-se observar para 0 dias, que a adição da moringa no PLA promoveu uma pequena perda de estabilidade quando comparado com o PLA puro. Após 160 dias, o biocompósito PLA/MO apresentou uma perda de estabilidade térmica significativa e equivalente aquela observada no PLA puro. A redução na estabilidade térmica do PLA, decorreu da elevada absorção de água pela moringa em pó, acelerando o processo de hidrólise do PLA, com consequente redução de sua massa molar.

Na Tabela 3 podem ser observados os valores correspondentes aos principais eventos térmicos do PLA, das misturas poliméricas PLA/SEBS e dos seus biocompósitos com moringa. O PLA apresentou apenas um evento térmico degradativo à $348,87{ }^{\circ} \mathrm{C}$ e as misturas com SEBS apresentaram dois eventos: um em torno de $340{ }^{\circ} \mathrm{C}$ e outro em torno de $405{ }^{\circ} \mathrm{C}$, correspondente à degradação do PLA e do SEBS, respectivamente. Isso mostra que o SEBS é mais estável termicamente do que o PLA. Foi observada uma $\mathrm{T}_{\text {inicial }}$ de $348^{\circ} \mathrm{C}, 344^{\circ} \mathrm{C}, 338^{\circ} \mathrm{C}, 335^{\circ} \mathrm{C}, 326^{\circ} \mathrm{C}$ e $320^{\circ} \mathrm{C}$ para as composições de PLA, PLA/SEBS 90/10, PLA/SEBS 70/30, PLA/MO 100/1, PLA/SEBS/MO 90/10/1 e PLA/SEBS/MO 70/30/1, respectivamente. Dessa forma, pode-se perceber que a adição do SEBS e da moringa, reduziu a estabilidade térmica do PLA, porque o SEBS pode ter inibido o crescimento do cristal de PLA (Tsou et al., 2015). 
Research, Society and Development, v. 10, n. 4, e12210413249, 2021

(CC BY 4.0) | ISSN 2525-3409 | DOI: http://dx.doi.org/10.33448/rsd-v10i4.13249

Tabela 3 - Valores de $\mathrm{T}_{\text {inicial }}, \mathrm{T}_{\text {máximo, }}, \mathrm{T}_{10 \%}, \mathrm{~T}_{50 \%}$ e $\mathrm{T}_{\text {final }}$ das blendas PLA/SEBS e dos seus compósitos com moringa oleífera.

\begin{tabular}{|c|c|c|c|c|c|c|c|c|c|}
\hline \multirow{2}{*}{ Tempo de exposição } & \multirow{2}{*}{ Composições } & \multicolumn{2}{|c|}{$\mathrm{T}_{\text {inicial }}\left({ }^{\circ} \mathrm{C}\right)$} & \multicolumn{2}{|c|}{$\mathrm{T}_{\text {máxima }}\left({ }^{\circ} \mathrm{C}\right)$} & \multirow{2}{*}{$\mathrm{T}_{10 \%}\left({ }^{\circ} \mathrm{C}\right)$} & \multirow{2}{*}{$\mathrm{T}_{50 \%}\left({ }^{\circ} \mathrm{C}\right)$} & \multicolumn{2}{|c|}{$\mathrm{T}_{\text {final }}\left({ }^{\circ} \mathrm{C}\right)$} \\
\hline & & Evento 1 & Evento 2 & Evento 1 & Evento 2 & & & Evento 1 & Evento 2 \\
\hline \multirow{7}{*}{0 dias } & PLA & 348,87 & - & 387,33 & - & 355,70 & 379,72 & 398,52 & - \\
\hline & Moringa & 404,89 & - & 445,31 & - & 417,44 & 442,19 & 472,72 & - \\
\hline & PLA/SEBS 90/10 & 344,27 & 398,29 & 378,49 & 352,71 & 345,70 & 372,77 & 389,16 & 481,22 \\
\hline & PLA/SEBS 70/30 & 338,38 & 416,96 & 377,08 & 458,19 & 348,01 & 374,68 & 386,21 & 480,99 \\
\hline & PLA/MO 100/1* & 335,44 & - & 375,73 & - & 342,53 & 368,18 & 388,74 & - \\
\hline & PLA/SEBS/MO /90/10/1* & 326,07 & 406,06 & 369,60 & 448,88 & 332,68 & 360,27 & 376,67 & 478,51 \\
\hline & PLA/SEBS/MO /70/30/1* & 320,83 & 419,25 & 362,06 & 459,60 & 335,46 & 362,86 & 373,78 & 482,58 \\
\hline \multirow{7}{*}{160 dias } & PLA & 286,85 & - & 335,87 & & 296,01 & 325,86 & 347,83 & - \\
\hline & Moringa & 404,89 & - & 445,31 & - & 417,44 & 442,19 & 472,72 & - \\
\hline & PLA/SEBS 90/10 & 293,94 & 402,74 & 337,70 & 441,25 & 302,14 & 330,49 & 348,14 & 465,31 \\
\hline & PLA/SEBS 70/30 & 312,44 & 409,52 & 344,16 & 448,65 & 312,90 & 345,37 & 355,88 & 470,25 \\
\hline & PLA/MO 100/1* & 299,12 & - & 341,05 & - & 301,27 & 333,77 & 356,19 & - \\
\hline & PLA/SEBS/MO 90/10/1* & 305,90 & 402,74 & 342,62 & 442,20 & 306,28 & 335,48 & 352,21 & 466,27 \\
\hline & PLA/SEBS/MO 70/30/1* & 314,27 & 409,20 & 340,73 & 448,97 & 317,46 & 345,03 & 356,86 & 473,04 \\
\hline
\end{tabular}

Fonte: Autores. 


\subsection{Biodegradação em ambiente marinho simulado}

A Figura 3 mostra as curvas de variação de massa em função do tempo de exposição ao ambiente marinho simulado para as composições avaliadas. Pode-se observar que as blendas com 10 e 30\% de SEBS apresentaram uma taxa de biodegradação mais acelerada do que o PLA puro, para os tempos de 40 e 80 dias de exposição. Isso pode ter ocorrido devido ao fato do PLA puro ter tido um aumento de cristalinidade mais significativo do que aquele observado no PLA presente nas blendas, como observado por meio da Tabela 2. As composições PLA/SEBS/MO 90/10/1 e PLA/SEBS/MO 70/30/1 tiveram um ganho de massa (0.69 \% e 3.77\%), seguido por uma redução (0\% e $1.07 \%)$, e por um aumento $(0.66 \%$ e $2.09 \%$ ), para os tempos de 40, 80 e 160 dias, respectivamente. Esse ganho de massa inicial pode estar relacionado à natureza hidrofílica das partículas de moringa em pó, a qual pode ter levado à absorção de água através dos poros do biocompósito (Gunti. et al, 2018). Contudo, é interessante observar que a composição PLA/MO 100/1, apresentou taxa de degradação superior aquela reportada no PLA puro, para os tempos de 40 e 80 dias de exposição. Isso já era esperado pois como citado anteriormente a cristalinidade da composição PLA/MO 100/1 foi cerca até $11 \%$ menor do que aquela observada no PLA, para um mesmo tempo de exposição (Figura 2). Com mais regiões amorfas disponíveis, as ligações éster do PLA foram atacadas mais facilmente por moléculas de água, resultando na liberação de oligômeros de ácido lático (Iovino et al., 2008; Paul et al., 2005). Para o tempo de 160 dias em ambiente marinho simulado, apenas 3 composições apresentaram perda de massa, onde as amostras de PLA apresentaram uma taxa de degradação equivalente à composição PLA/SEBS 90/10, e a composição PLA/SEBS 70/30 uma perda de massa um pouco menor.

Figura 4 - Variação de massa das blendas PLA/SEBS e dos seus compósitos com moringa

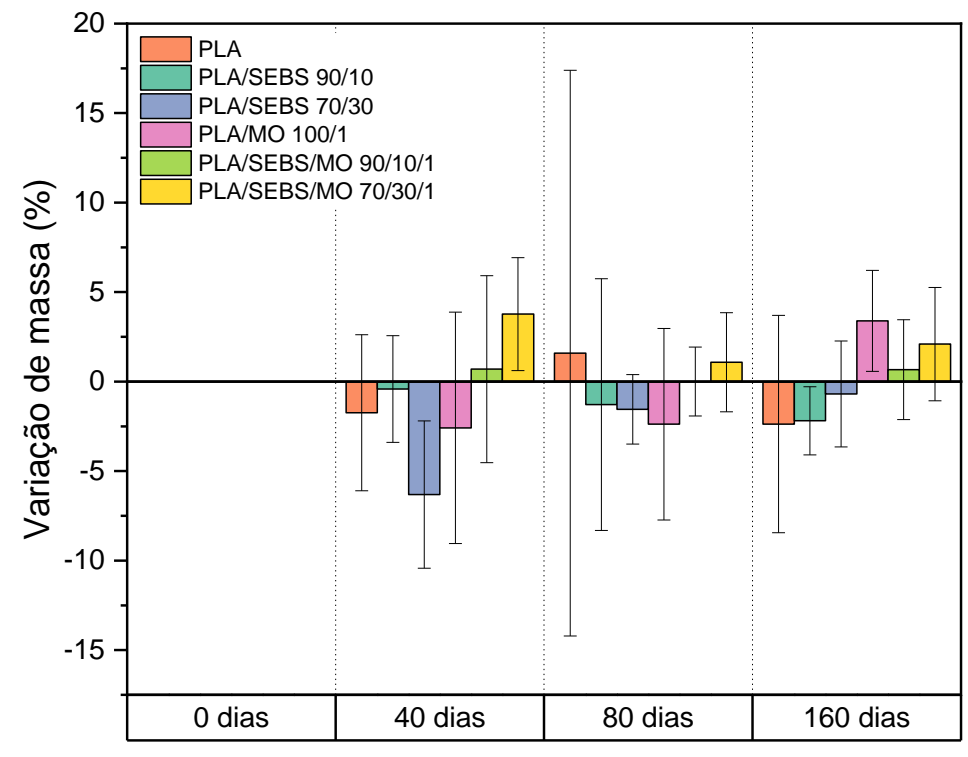

Fonte: Autores.

\section{Conclusão}

Os biocompósitos à base das misturas de PLA/SEBS e PLA/SEBS/MO com PLA como o componente principal foram fabricados por meio de um processo de mistura por fusão. As transições térmicas e os comportamentos de decomposição térmica foram investigados por um calorímetro diferencial de varredura e por um analisador termogravimétrico, 
Research, Society and Development, v. 10, n. 4, e12210413249, 2021

(CC BY 4.0) | ISSN 2525-3409 | DOI: http://dx.doi.org/10.33448/rsd-v10i4.13249

respectivamente. A água absorvida pela moringa, reduziu a estabilidade térmica do PLA, sugerindo que houve uma degradação mais acelerada para essas composições devido à hidrólise das cadeias de PLA. As análises de DSC mostraram houve aumento da fração cristalina com o aumento do tempo de exposição, em decorrência do maior consumo das regiões amorfas. A avaliação da variação de massa mostrou que o SEBS parece não ter afetado a biodegradação do PLA, pois mesmo após 160 dias de exposição não foram observadas taxas de degradações maiores nas blendas PLA/SEBS. Além disso, as composições com SEBS e Moringa, não apresentaram perda de massa, apenas ganho. Isso significa que para as condições estudadas não pode-se afirmar que essas misturas são biodegradáveis.

Como sugestão para trabalhos futuros existe a possibilidade de se utilizar um agente compatibilizante para melhorar a interação entre as matrizes de PLA e SEBS, de forma a amplificar a estabilidade térmica da blenda PLA/SEBS. Além disso também é interessante variar as condições de exposição em ambiente marinho, como por exemplo: temperatura, pressão, quantidade de oxigênio, e salinidade.

\section{Referências}

Abdelwahab, M. A., Flynn, A., Chiou, B.-S., Imam, S., Orts, W., \& Chiellini, E. (2012). Thermal, mechanical and morphological characterization of plasticized PLA-PHB blends. Polymer Degradation and Stability, 97(9), 1822-1828.

Alegbeleye, O. O. (2018). How functional is Moringa Oleifera? A review of its nutritive, medicinal, and socioeconomic potential. Food and Nutrition Bulletin, $39(1), 149-170$

Alias, N. F., \& Ismail, H. (2019). An overview of toughening polylactic acid by an elastomer. Polymer-Plastics Technology and Materials, 58(13), 13991422 .

Balakrishnan, H., Hassan, A., \& Wahit, M. U. (2010). Mechanical, thermal, and morphological properties of polylactic acid/linear low density polyethylene blends. Journal of Elastomers \& Plastics, 42(3), 223-239.

Bernardes, G. P., da Rosa Luiz, N., Santana, R. M. C., \& de Camargo Forte, M. M. (2019). Rheological behavior and morphological and interfacial properties of PLA/TPE blends. Journal of Applied Polymer Science, 136(38), 47962.

Capitain, C., Ross-Jones, J., Möhring, S., \& Tippkötter, N. (2020). Differential scanning calorimetry for quantification of polymer biodegradability in compost. International Biodeterioration \& Biodegradation, 149, 104914.

Carrasco, F., \& Pagès, P. (2008). Thermal degradation and stability of epoxy nanocomposites: Influence of montmorillonite content and cure temperature. Polymer Degradation and Stability, 93(5), 1000-1007. https://doi.org/10.1016/j.polymdegradstab.2008.01.018

Carrasco, F., Pagès, P., Gámez-Pérez, J., Santana, O. O., \& Maspoch, M. L. (2010). Processing of poly(lactic acid): Characterization of chemical structure, thermal stability and mechanical properties. Polymer Degradation and Stability, 95(2), 116-125.https://doi.org/10.1016/j.polymdegradstab.2009.11.045

Chang, J., An, Y. U., \& Sur, G. S. (2003). Poly (lactic acid) nanocomposites with various organoclays. I. Thermomechanical properties, morphology, and gas permeability. Journal of Polymer Science Part B: Polymer Physics, 41(1), 94-103.

Chow, W. S., Tham, W. L., Poh, B. T., \& Ishak, Z. A. M. (2018). Mechanical and thermal oxidation behavior of poly (Lactic Acid)/halloysite nanotube nanocomposites containing N, N'-Ethylenebis (Stearamide) and SEBS-g-MA. Journal of Polymers and the Environment, 26(7), $2973-2982$.

Dhar, P., \& Katiyar, V. (2017). Thermal degradation kinetics of polylactic acid/acid fabricated cellulose nanocrystal based bionanocomposites. International Journal of Biological Macromolecules, 104, 827-836.

Dias, P. D. P., \& Chinelatto, M. A. (2019). Effect of poly ( $\varepsilon$-caprolactone-b-tetrahydrofuran) triblock copolymer concentration on morphological, thermal and mechanical properties of immiscible PLA/PCL blends. Journal of Renewable Materials, 7(2), 129-138.

Falowo, A. B., Mukumbo, F. E., Idamokoro, E. M., Lorenzo, J. M., Afolayan, A. J., \& Muchenje, V. (2018). Multi-functional application of Moringa oleifera Lam. in nutrition and animal food products: A review. Food Research International, 106, 317-334.

Fortunati, E., Puglia, D., Kenny, J. M., Haque, M. M.-U., \& Pracella, M. (2013). Effect of ethylene-co-vinyl acetate-glycidylmethacrylate and cellulose microfibers on the thermal, rheological and biodegradation properties of poly (lactic acid) based systems. Polymer Degradation and Stability, 98(12), 27422751.

Gabbott, P. (2008). Principles and applications of thermal analysis. John Wiley \& Sons.

George, A., Sanjay, M. R., Srisuk, R., Parameswaranpillai, J., \& Siengchin, S. (2020). A comprehensive review on chemical properties and applications of biopolymers and their composites. International Journal of Biological Macromolecules, 154, 329-338. 
Research, Society and Development, v. 10, n. 4, e12210413249, 2021

(CC BY 4.0) | ISSN 2525-3409 | DOI: http://dx.doi.org/10.33448/rsd-v10i4.13249

Gunti, R., Ratna Prasad, A. V, \& Gupta, A. (2018). Mechanical and degradation properties of natural fiber-reinforced PLA composites: Jute, sisal, and elephant grass. Polymer Composites, 39(4), 1125-1136.

Gupta, S., Jain, R., Kachhwaha, S., \& Kothari, S. L. (2018). Nutritional and medicinal applications of Moringa oleifera Lam.-Review of current status and future possibilities. Journal of Herbal Medicine, 11, 1-11.

Hakim, R. H., Cailloux, J., Santana, O. O., Bou, J., Sánchez-Soto, M., Odent, J., Raquez, J.-M., Dubois, P., Carrasco, F., \& Maspoch, M. L. (2017). PLA/SiO2 composites: Influence of the filler modifications on the morphology, crystallization behavior, and mechanical properties. Journal of Applied Polymer Science, 134(40), 45367.

Haque, M. M.-U., Alvarez, V., Paci, M., \& Pracella, M. (2011). Processing, compatibilization and properties of ternary composites of Mater-Bi with polyolefins and hemp fibres. Composites Part A: Applied Science and Manufacturing, 42(12), 2060-2069.

Iovino, R., Zullo, R., Rao, M. A., Cassar, L., \& Gianfreda, L. (2008). Biodegradation of poly (lactic acid)/starch/coir biocomposites under controlled composting conditions. Polymer Degradation and Stability, 93(1), 147-157.

Jia, C., Lu, P., \& Zhang, M. (2020). Preparation and Characterization of Environmentally Friendly Controlled Release Fertilizers Coated by Leftovers-Based Polymer. Processes, 8(4), 417.

Jose, S., Thomas, S., Lievana, E., \& Karger-Kocsis, J. (2005). Morphology and mechanical properties of polyamide 12 blends with styrene/ethylenebutylene/styrene rubbers with and without maleation. Journal of Applied Polymer Science, 95(6), 1376-1387.

Juárez, D., Ferrand, S., Fenollar, O., Fombuena, V., \& Balart, R. (2011). Improvement of thermal inertia of styrene-ethylene/butylene-styrene (SEBS) polymers by addition of microencapsulated phase change materials (PCMs). European Polymer Journal, 47(2), 153-161.

Krishnan, S., Mohanty, S., \& Nayak, S. K. (2018). An eco-friendly approach for toughening of polylactic acid from itaconic acid based elastomer. Journal of Polymer Research, 25(1), 1-8.

Li, D., Shentu, B., \& Weng, Z. (2011). Morphology, rheology, and mechanical properties of polylactide/poly (ethylene-co-octene) blends. Journal of Macromolecular Science, Part B, 50(10), 2050-2059.

Lima, J. C. C., Araújo, E. A. G., Agrawal, P., \& Mélo, T. J. A. (2019). PLA/SEBS Bioblends: Influence of SEBS Content and of Thermal Treatment on the Impact Strength and Morphology. Macromolecular Symposia, 383(1), 1700072. https://doi.org/10.1002/masy.201700072

Liu, Y., Wang, X., Wei, X., Gao, Z., \& Han, J. (2018). Values, properties and utility of different parts of Moringa oleifera: An overview. Chinese Herbal Medicines, 10(4), 371-378.

Merino, D., Mansilla, A. Y., Casalongué, C. A., \& Alvarez, V. A. (2019). Performance of bio-based polymeric agricultural mulch films. In Polymers for Agrifood applications (pp. 215-240). Springer.

Mishra, K., \& Sinha, S. (2020). Development and assessment of Moringa oleifera (Sahajana) leaves filler/epoxy composites: Characterization, barrier properties and in situ determination of activation energy. Polymer Composites, 41(12), 5016-5029.

Mittal, A., Garg, S., \& Bajpai, S. (2020). Thermal decomposition kinetics and properties of grafted barley husk reinforced PVA/starch composite films for packaging applications. Carbohydrate Polymers, 240, 116225.

Muniyasamy, S., Ofosu, O., John, M. J., \& Anandjiwala, R. D. (2016). Mineralization of poly (lactic acid)(PLA), poly (3-hydroxybutyrate-covalerate)(PHBV) and PLA/PHBV blend in compost and soil environments. Journal of Renewable Materials, 4(2), 133-145.

Nehra, R., Maiti, S. N., \& Jacob, J. (2018a). Analytical interpretations of static and dynamic mechanical properties of thermoplastic elastomer toughened PLA blends. Journal of Applied Polymer Science, 135(1), 1-13. https://doi.org/10.1002/app.45644

Nehra, R., Maiti, S. N., \& Jacob, J. (2018b). Effect of Thermoplastic Elastomer on Melt Rheological and Fracture Behavior of Poly (Lactic Acid). PolymerPlastics Technology and Engineering, 57(12), 1254-1264.

Palsikowski, P. A., Kuchnier, C. N., Pinheiro, I. F., \& Morales, A. R. (2018). Biodegradation in soil of PLA/PBAT blends compatibilized with chain extender. Journal of Polymers and the Environment, 26(1), 330-341.

Park, S.-B., Lih, E., Park, K.-S., Joung, Y. K., \& Han, D. K. (2017). Biopolymer-based functional composites for medical applications. Progress in Polymer Science, 68, 77-105.

Paul, M.-A., Delcourt, C., Alexandre, M., Degée, P., Monteverde, F., \& Dubois, P. (2005). Polylactide/montmorillonite nanocomposites: study of the hydrolytic degradation. Polymer Degradation and Stability, 87(3), 535-542.

Pelegrini, K., Donazzolo, I., Brambilla, V., Coulon Grisa, A. M., Piazza, D., Zattera, A. J., \& Brandalise, R. N. (2016). Degradation of PLA and PLA in composites with triacetin and buriti fiber after 600 days in a simulated marine environment. Journal of Applied Polymer Science, 133(15), n/a-n/a. https://doi.org/10.1002/app.43290

Pereira, A. S., Shitsuka, D. M., Parreira, F. J., \& Shitsuka, R. (2018). Metodologia da pesquisa científica.

Pérez, I. P., Pasandín, A. M. R., Pais, J. C., \& Pereira, P. A. A. (2019). Use of lignin biopolymer from industrial waste as bitumen extender for asphalt mixtures. Journal of Cleaner Production, 220, 87-98. 
Research, Society and Development, v. 10, n. 4, e12210413249, 2021

(CC BY 4.0) | ISSN 2525-3409 | DOI: http://dx.doi.org/10.33448/rsd-v10i4.13249

Pollet, E., Paul, M.-A., \& Dubois, P. (2003). New aliphatic polyester layered-silicate nanocomposites. In Biodegradable polymers and plastics (pp. 327-350). Springer.

Qi, R., Luo, M., \& Huang, M. (2011). Synthesis of styrene-ethylene-butylene-styrene triblock copolymer-g-polylactic acid copolymer and its potential application as a toughener for polylactic acid. Journal of Applied Polymer Science, 120(5), 2699-2706.

Ramsay, B. A., Langlade, V., Carreau, P. J., \& Ramsay, J. A. (1993). Biodegradability and mechanical properties of poly-(beta-hydroxybutyrate-co-betahydroxyvalerate)-starch blends. Applied and Environmental Microbiology, 59(4), 1242-1246.

Sanjay, M. R., Madhu, P., Jawaid, M., Senthamaraikannan, P., Senthil, S., \& Pradeep, S. (2018). Characterization and properties of natural fiber polymer composites: A comprehensive review. Journal of Cleaner Production, 172, 566-581.

Soni, R., Kapri, A., Zaidi, M. G. H., \& Goel, R. (2009). Comparative biodegradation studies of non-poronized and poronized LDPE using indigenous microbial consortium. Journal of Polymers and the Environment, 17(4), 233.

Tejada-Oliveros, R., Balart, R., Ivorra-Martinez, J., Gomez-Caturla, J., Montanes, N., \& Quiles-Carrillo, L. (2021). Improvement of Impact Strength of Polylactide Blends with a Thermoplastic Elastomer Compatibilized with Biobased Maleinized Linseed Oil for Applications in Rigid Packaging. Molecules, $26(1), 240$.

Tsou, C.-H., Kao, B.-J., Yang, M.-C., Suen, M.-C., Lee, Y.-H., Chen, J.-C., Yao, W.-H., Lin, S.-M., Tsou, C.-Y., Huang, S.-H., De Guzman, M., \& Hung, W.S. (2015). Biocompatibility and characterization of polylactic acid/styrene-ethylene-butylene-styrene composites. Bio-Medical Materials and Engineering, 26(s1), S147-S154. https://doi.org/10.3233/BME-151300

Valenga, M. G. P., Boschen, N. L., Rodrigues, P. R. P., \& Maia, G. A. R. (2019). Agro-industrial waste and Moringa oleifera leaves as antioxidants for biodiesel. Industrial Crops and Products, 128, 331-337.

Verma, D., Fortunati, E., Jain, S., \& Zhang, X. (2019). Biomass, Biopolymer-Based Materials, and Bioenergy: Construction, Biomedical, and Other Industrial Applications. Woodhead Publishing.

Vigneshwaran, S., Sundarakannan, R., John, K. M., Johnson, R. D. J., Prasath, K. A., Ajith, S., Arumugaprabu, V., \& Uthayakumar, M. (2020). Recent advancement in the natural fiber polymer composites: a comprehensive review. Journal of Cleaner Production, 124109.

Wang, Y., Wei, Z., \& Li, Y. (2018). Toughening polylactide with epoxidized styrene-butadiene impact resin: Mechanical, morphological, and rheological characterization. Journal of Applied Polymer Science, 135(13), 1-8. https://doi.org/10.1002/app.46058

Wilkinson, A. N., Clemens, M. L., \& Harding, V. M. (2004). The effects of SEBS-g-maleic anhydride reaction on the morphology and properties of polypropylene/PA6/SEBS ternary blends. Polymer, 45(15), 5239-5249.

Yahya, E. B., Jummaat, F., Amirul, A. A., Adnan, A. S., Olaiya, N. G., Abdullah, C. K., Rizal, S., Mohamad Haafiz, M. K., \& Khalil, H. P. S. (2020). A review on revolutionary natural biopolymer-based aerogels for antibacterial delivery. Antibiotics, 9(10), 648.

Yan, J., \& Spontak, R. J. (2019). Toughening Poly (lactic acid) with Thermoplastic Elastomers Modified by Thiol-ene Click Chemistry. ACS Sustainable Chemistry \& Engineering, 7(12), 10830-10839.

Zhang, G., Zhang, J., Zhou, X., \& Shen, D. (2003). Miscibility and phase structure of binary blends of polylactide and poly (vinylpyrrolidone). Journal of Applied Polymer Science, 88(4), 973-979. 